\title{
¿Cómo son las comunidades marginales que generan pobreza estructural?
}

\section{What do marginal communities that generate structural poverty look like?}

\author{
Susana Herrero-Olarte
}

Universidad de Las Américas (UDLA) en Quito, Ecuador

\section{Resumen}

En esta investigación se pretende comprobar si las comunidades marginales se definen como aquéllas en el ámbito rural de países de ingreso medio y bajo localizadas en zonas poco aptas para la agricultura con elevadas tasas de dispersión poblacional. El análisis comparativo de los indicadores de pobreza entre las comunidades y los promedios nacionales permite concluir que la definición propuesta es la adecuada. Los niveles de pobreza registrados ameritan intervenciones específicas e inminentes por parte de los titulares de obligaciones capaces de modificar las condiciones que llevan enquistando una pobreza que es ya estructural. La estrategia podría apoyarse en una propuesta de intervención regional o global por la similitud entre los indicadores de las comunidades, con condiciones de vidas similares, capaz de atender las principales necesidades detectadas.

Palabras clave: Desarrollo rural, marginación, comunidades marginadas, erradicación de pobreza, pobreza cuantitativa y cualitativa.

\section{Abstract}

The aim of this research was to determine whether marginal comunities could be defined as such in the rural areas of low- and medium-income countries with highly scattered populations. A comparative analysis of poverty indicators between such communities and national averages made it possible to conclude that the proposed definite was indeed appropriate. The poverty levels recorded call for specific, immediate interventions by those responsible, in order to modify the conditions that have led to what is now structural poverty. Given the similarities in living conditions and other indicators for these communities, the response strategy could rely on a proposal of either regional or global intervention geared to addressing the main needs detected.

Keywords: Rural development, marginalization, marginalized communities, eradication of poverty, quantitative and qualitative poverty. 


\section{INTRODUCCIÓN}

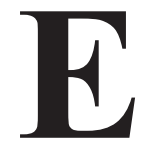

1 análisis de la pobreza se ha concentrado históricamente en la falta de recursos económicos, lo que impedía comprar los bienes capaces de satisfacer necesidades básicas individuales y familiares, definición general de pobreza cuantitativa. Eran pobres los que tenían unos ingresos inferiores a un parámetro previamente definido. El posterior análisis derivó en la pobreza cualitativa, en alusión a las necesidades no cubiertas. Eran pobres los que no tenían condiciones óptimas de salud, educación, etc.

Tanto al considerar la pobreza cuantitativa como cualitativa el reto es tratar de reducirla en el corto plazo y eliminarla en el largo a través de políticas diseñadas y desarrolladas por los titulares de obligaciones. Deben estar adaptadas a los diferentes contextos en los que se genera la pobreza para que resulten eficaces, puedan generar el impacto requerido y sean viables y sostenibles en el tiempo.

Del estudio de la desigualdad y las necesidades humanas no cubiertas surgen los conceptos de marginación y exclusión, que llaman la atención sobre las condiciones de vida que se dan en ciertos lugares y que irremediablemente producen pobreza.

Esta investigación pretende identificar las características de las comunidades marginales para poder localizarlas y conocerlas, e invitar a los titulares de obligaciones relacionados a diseñar y aplicar políticas para erradicar la pobreza que generan.

Las comunidades marginales se localizan en el ámbito rural de los países de ingreso medio y bajo donde, en términos generales, se registran los peores indicadores en términos de pobreza cuantitativa y cualitativa a nivel nacional, regional y mundial. Dado que la agricultura es una de las fuentes de ingresos fundamental, si las condiciones climáticas y del entorno limitan la productividad, se dificultará generar ingresos y por tanto superar la pobreza cuantitativa. Además, si se trata de comunidades con un elevado índice de dispersión recibirán una menor inversión por parte de las autoridades y tendrán menos acceso a servicios públicos, lo que se traduce en un peor dato de pobreza cualitativa. La menor inversión responde a la poca rentabilidad para la clase política porque su resultado se traduciría en una cantidad mínima de votos por las pocas personas que podrían beneficiarse por la condición de dispersión. 
Según lo anterior las comunidades marginadas son aquéllas en países de ingreso medio y bajo en el ámbito rural ubicadas en zonas poco aptas para la agricultura y en condición de dispersión.

Para conocer los datos de pobreza cuantitativa y cualitativa de las comunidades marginadas con la definición presentada y dada la poca información secundaria disponible se ha acudido a la información primaria obtenida en 53 comunidades marginales de 11 países de ingreso medio y bajo. Los resultados de las entrevistas y las encuestas realizadas a las familias que vivían en las comunidades y a los directivos y técnicos de las Organizaciones No Gubernamentales (ONG) que trabajan en este tipo de comunidades se compararon con los promedios nacionales.

En todos los casos los datos registrados en las comunidades eran peores a los promedios país. La diferencia en el caso de los indicadores de pobreza cuantitativa era de 56 por ciento y en el caso de la cualitativa de 35 por ciento Las generaciones pasadas presentaban, según las entrevistas mantenidas, indicadores muy similares. Los datos entre comunidades han sido muy parecidos, con un promedio de cinco por ciento de diferencia entre los mejores y los peores indicadores registrados.

\section{POBREZA Y MARGINACIÓN: CONCEPTOS Y CARACTERIZACIÓN}

El estudio de la pobreza ocupa parte del trabajo de economistas clásicos, como Smith (Heilbroner, 1976), Ricardo (1986, editado por Sraffa) o Matlhus (Malthus, 1846), entendida de manera general como el nivel de consumo que no asegura la subsistencia. Booth (1889) realizaron los primeros análisis cuantitativos comparados en el tiempo. Merton (1938), Stouffer et al. (1949), Merton et al. (1950) y Davis (1959), desde la sociología, profundizaron en la idea de las variaciones en la privación percibida de un individuo respecto al grupo. Runciman (1966) trasladó los avances en la teoría de la privación al ámbito económico, entendida como la imposibilidad de disponer de "algo" que sí puede tener parte o todo el grupo al que pertenece el individuo. La definición de este "algo", generó dos corrientes de pensamiento que dieron lugar al estudio de la pobreza cuantitativa, como se había entendido hasta el momento, y de la cualitativa, que pese a que había sido esbozada con anterioridad, cobró fuerza en adelante. Además resultó determinante la definición de las necesidades que debían satisfacer los individuos para determinar ese "algo" que debía cubrirlas. 
En lo que se conocería después como el enfoque economicista dominante de la pobreza ${ }^{1}$ o pobreza cuantitativa, algunos autores optaron por considerar que ese "algo" que una persona no tenía y otros sí era dinero. Las necesidades que debían cubrir eran, de manera general, las más básicas relacionadas con la subsistencia, y especialmente con la alimentación. Sus análisis derivaron en varios sistemas para establecer los límites que definen la pobreza, si bien los más conocidos y utilizados son el de la pobreza absoluta y el de la relativa. La pobreza absoluta es la cantidad mínima de ingresos diarios necesarios para subsistir, mientras la pobreza relativa establece un mínimo capaz de comprar la canasta básica de bienes y servicios nacional o regional. Tradicionalmente organismos como el Banco Mundial (Banco Mundial, 2016) o la Unión Europea se han apoyado en el estudio de la pobreza absoluta; mientras que la Comisión Económica para América Latina y el Caribe (CEPAL), la Agencia Central de Inteligencia (CIA, por sus siglas en inglés) o el Programa de las Naciones Unidas para el Desarrollo (PNUD) aplican más datos de la pobreza relativa. No obstante, los distintos organismos hacen cada vez más referencia a ambas metodologías, de cara a alcanzar una visión comparada global más integral y real de la pobreza.

Otros analistas, en cambio, sentaban las bases del conocimiento de la pobreza cualitativa al considerar que ese "algo" que les hacía falta las personas consideradas pobres no era necesariamente dinero, apoyándose en los estudios empíricos que mostraban la relación inversa entre ingresos y la calidad de vida (Streeten et al., 1981). El estudio sobre los distintos tipos de necesidades y prioridades del individuo se reforzaba tras los estudios que cuestionaban la existencia del hombre racional, maximizador, con conocimiento y previsión perfecta (Thaler, 1988 o Henrich et al., 2005), poniéndose en duda especialmente que primara su necesidad de buscar siempre el beneficio económico e individual. El estudio de las necesidades humanas continuó desarrollándose desde las ciencias sociales (Maslow, 1943; Fromm, 1956; Wiggins et al., 2002), recuperándose tímidamente desde la economía el trabajo al respecto de Karl Marx (Márkus, 1974; Heller, 1978), y habiendo de destacar la aportación de Townsend (1979), que apuntalaba la generación de nueva teoría, al equiparar las necesidades humanas a la participación en los patrones ordinarios de vida, como la dieta o el modo de vestir. Glaser y Strauss (1967) sistematizaron los primeros modelos al respecto.

${ }^{1}$ Yitzhaki (1979, 1982), Altimir (1979), Hey y Lambert (1980), Berrebi y Silber (1985), Podder (1996), Ebert y Moyes (2000), Bárcena (2001), Mukherjee (1997), Ravallion (1998), Imedio y Bárcena (2002), Chakravarty y Mukherjee (1998) y Chakravarty y Moyes (2002). 
Profundizando en el concepto de pobreza cualitativa, resultó definitivo y un referente en el tema el trabajo de Sen, economista reconocido además por oscilar entre la sociología y la filosofía, disciplinas fundamentales para el desarrollo del pensamiento entorno a la pobreza cualitativa. El autor consideraba que era necesario que existiesen los bienes y servicios pero que era más importante que las personas pudieran acceder a ellos, es decir, que tengan la titularidad o el derecho a consumirlos, almacenarlos, etc. Una vez tiene la titularidad, el sujeto debe poder ser capaz de hacerla funcionar. Los funcionamientos son los estados en los que se encuentra el sujeto por poder disponer de una titularidad, lo que nos indicaría su nivel de bienestar ${ }^{2}$ (Sen, 1982, 1984, 1985). La pobreza es por tanto para Sen (1990) el estado en el que son negadas a la persona las opciones, es decir, le limitan la capacidad para tener ser y hacer lo que quiera en la vida. Se le reducen por tanto los funcionamientos y la manera de alcanzarlos, equiparando libertades con capacidades (Sen, 1999).

Entrar a valorar las distintas opciones del individuo genera un sinfín de combinaciones de indicadores para considerar la pobreza desde un punto de vista cualitativo o multidimensional. De hecho, se vuelve un concepto subjetivo que se condiciona por la propia percepción del individuo, $\mathrm{y}$ que depende además de su entorno (Wiggins, 2002), convirtiéndose en una cuestión dinámica, con unos indicadores difíciles de seleccionar y especialmente de medir. Destacan entre los indicadores de referencia el Índice de Pobreza Humana (IPH), creado por el PNUD en 1997, que valora la esperanza de vida, la alfabetización, el acceso al agua, y la nutrición; y el Índice de Pobreza Multidimensional (IPM), presentado en 2010 por el PNUD y la Oxford Poverty and Human Development Initiative (OPHI) que incluye parámetros de educación, salud y de tenencia de bienes materiales (Kovacevic y Calderon, 2014).

Cada vez más, el estudio de la pobreza cualitativa o multidimensional ${ }^{3}$ se ha visto complementado con el trabajo cuantitativo hasta generar sistemas de medición capaces de utilizar las herramientas de ambos sistemas, para ofrecer datos que reflejan menor la realidad. En su evolución, la pobreza cuantitativa y cualitativa han confluido en indicadores como el IPH-

\footnotetext{
${ }^{2}$ Por ejemplo, una persona puede tener un bocadillo en sus manos que es de ella. Puede comérselo, tirarlo, etc. es decir, tiene la titularidad sobre el bocadillo. Además, es capaz de realizar el funcionamiento de comérselo, si le gusta, lo que la situará en una situación de satisfacción porque ya no tiene hambre, mejorando su nivel de bienestar. Si no tuviera el bocadillo no tendría la titularidad y por tanto no podría comérselo, sin dientes no sería capaz de masticarlo, y si fuera alérgico al pan, el funcionamiento de comérselo para no tener hambre, disminuiría su bienestar. ${ }^{3}$ Habiendo de destacar el trabajo de Pradhan y Ravailllon (2000), Bamberger (2000), y Bowen (2009).
} 
2, una evolución del primero que incluía ingresos per cápita por familia; y en conceptos íntimamente relacionados, como el de la exclusión social y el de la marginación.

Al considerar las variables que llevan a un individuo a la condición de pobreza cuantitativa o cualitativa, es preciso atender a los conceptos de marginación y exclusión.

Desde la sociología se vinculaba la marginación con la migración como la falta de adaptación o pertenencia cultural (Thomas y Znaniecki, 1918 y Park, 1928). La atención se dirigió después también hacia los nacionales y a su también posible condición de marginación (Green, 1947-48 y Golovensky, 1951-52). En su relación con la economía, los marginados eran aquéllos que no generaban, de manera activa, redes productoras de riqueza y de reconocimiento social (Massé, 1965 y Lenoir, 1974), de las que nunca fueron parte (Geremek, 1991) o dejaron de serlo (Castel, 1998). Otras consideraciones de la marginación posicionaban al hombre como un ente pasivo, que no participaba de la evolución social del grupo (Desal, 1969), no incidía en sus problemas, y no cambiaba su realidad ni contaba con ningún órgano político que le representase (Giusti, 1973). El hombre era entonces discriminado porque no tenía igual trato que el resto de ciudadanos (UNESCO, 1960) o no tenía acceso a derechos universalmente reconocidos (Minujin, 1998), y se le utilizaba como reserva para presionar a la baja los salarios (Cardoso, 1970).

Surge de manera paralela la valoración que no considera la marginación como una característica del individuo y se la relaciona de manera directa con el lugar en el que vive. Una zona marginal es aquélla a la que no han llegado las estructuras, las normas o los valores de la modernidad. Es el lugar al que no han llegado todavía los beneficios del desarrollo, condicionando las oportunidades del individuo. Esta es la consideración del Consejo Nacional de Población (CONAPO) y el Programa de Educación, Salud y Alimentación (PROGRESA), que tratan de medir esos beneficios del desarrollo, las oportunidades y las libertades como el acceso a bienes y servicios básicos. Para el análisis, las organizaciones parten de indicadores locales de educación, vivienda e ingresos, y estatales de dispersión, para determinar qué comunidades se encuentran en condición de marginación, e invitar a las autoridades públicas a concentrar allí su trabajo. 
La definición de exclusión social difundida por la Unión Europea, ${ }^{4}$ atiende igualmente al lugar en el que está el sujeto, capaz de generar una estructura multifactorial y subjetiva que condiciona su estado.

La exclusión, que comparte rasgos fundamentales en su definición con la marginación, se concentra en el ámbito urbano mientras la marginación, cuestión en la que se profundiza en esta investigación, atiende especialmente al ámbito rural.

Para poder identificar a las comunidades marginales es preciso definir cuáles son los parámetros para considerarlas como tales, cuestión sobre la que pretende arrojar luz este trabajo. Los titulares de obligaciones podrán localizar los grupos que, por el lugar en que viven, tienen una dificultad estructural para salir de la pobreza y presentan los peores indicadores en el ámbito rural.

En esta investigación se estima que las comunidades marginales son grupos poblacionales localizadas en el ámbito rural de los países de ingreso medio y bajo, cuestión generalmente aceptada, y cumplen dos características: es muy difícil que las personas puedan generar ingresos y la inversión pública es muy escasa o nula.

Para que éstas sean dos características de las comunidades marginales deben darse tres condiciones. Los indicadores de pobreza cuantitativa y cualitativa deben ser sustancialmente peores a los promedios nacionales y deben haber sido similares o peores para las generaciones anteriores, lo que caracterizaría una cuestión estructural. Además, los indicadores deben ser similares entre las distintas comunidades del mundo, lo que invitaría a pensar que la condición de marginalidad genera resultados similares independientemente del lugar en el que se considere. Al final del documento se identifican las necesidades que presentan como prioritarias las familias en las comunidades, para iniciar el proceso de diseño de las estrategias a desarrollar.

La dificultad para generar ingresos se ha considerado una característica de las comunidades marginales porque limita la capacidad para mejorar indicadores de pobreza cualitativa. Dado que la principal fuente de ingresos es la producción primaria es necesario atender a la condición en la que se generan los menores ingresos de la producción agropecuaria. Olarte (2017) llamaba la atención sobre la falta de titularidad de la tierra y los lotes pequeños; sobre la falta de maquinaria y trabajadores; mientras señalaba la

\footnotetext{
${ }^{4}$ Habiendo de destacar a la aportación de Renes (1993), Abrahamson (1997), Tezanos (1999), Moreno (2000), Arriba (2002), Raya (2005).
} 
dificultad para comercializar la producción agropecuaria. Las tres son causas modificables con los recursos económicos y técnicos suficientes.

Lo que limita los ingresos de la producción agropecuaria y es difícil de superar son unas condiciones ambientales y climatológicas adversas, que impiden alcanzar niveles óptimos de productividad. Puede considerarse que tales condiciones dan en la selva o bosques, los desiertos, y las zonas congeladas, que como se aprecia en la Figura 1 suponen una parte sustancial de la Tierra. Habría que añadir cuestiones morfológicas del terreno, como las pendientes pronunciadas y planicies de altitud.

Para Sebastian (2009), 770 millones de personas vivían en zonas rurales poco aptas para la producción agropecuaria, lo que supondría 30 por ciento de la población del campo. El 65 por ciento se localizaban en Asia y 20 por ciento en África Subsahariana.

La segunda de las características de las comunidades marginales, además de la ubicación en territorios rurales y de que estos presenten bajos niveles de productividad agropecuaria, es la escasa densidad poblacional, lo que se asimila a una escasa inversión pública. Cuando la población es escasa, hacer una inversión pública no ofrece el rendimiento que las autoridades públicas están esperando, en la medida en que no se traduce en la cantidad de votos esperados. La falta de inversión pública, lo que se traduce en sistemas de salud y educación públicos deficientes, supone limitar las oportunidades para superar indicadores de pobreza cualitativa.

Sebastian (2009) equiparaba el estado de escasez de la población a la condición de aislamiento, que a su vez se equiparaba a la lejanía con el mercado más cercano. Para el autor, una zona estaba aislada si se localizaba a más de dos horas del mercado más cercano; de cuatro a ocho horas se consideraba aislamiento muy alto y a más de ocho horas, aislamiento extremo. Para Sebastian (2009) estaban en condición de marginación y aislamiento las dos terceras partes de los productores agropecuarios en países de renta media y baja, lo que suponían 1,700 millones de personas. Smael y Alpert (2009), con los mismos criterios, consideraban que el ser humano estaba abandonado por la naturaleza cuando estaba en una zona de escasa productividad agropecuaria, y abandonado por el ser humano cuando estaba lejos del mercado más cercano. Según sus estimaciones, 20 por ciento de la población agrícola de los países de renta baja y media, lo que suponía 542 millones de personas, estaba abandonado por la naturaleza y por el ser humano en 2008. 
¿Cómo son las comunidades marginales que generan pobreza estructural? /S. HERRERO-OLARTE

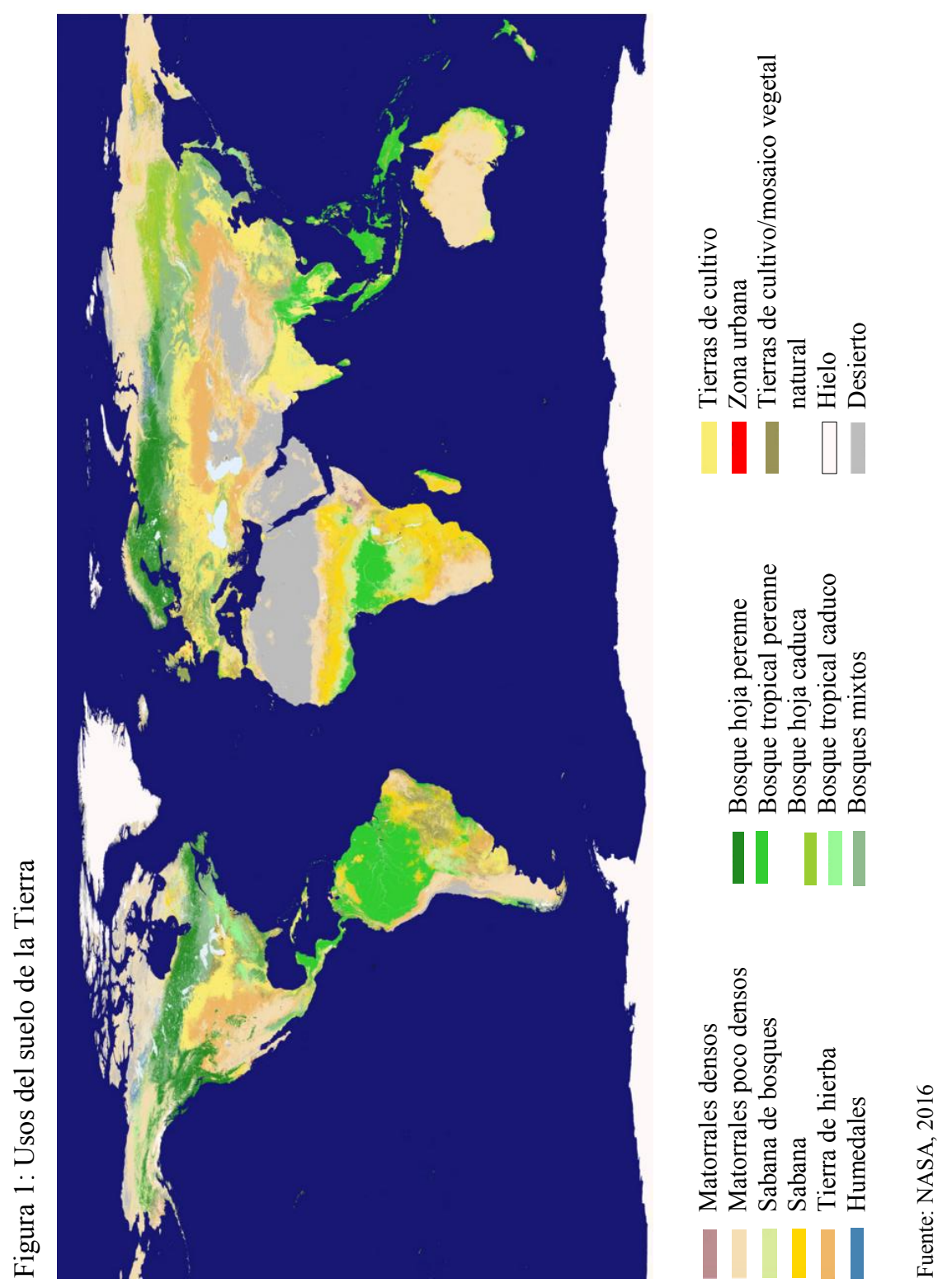


Asimilar la falta de inversión pública a la distancia al mercado más cercano implica que hay que medir cada caso de forma individual para comprobar si está lo suficientemente alejada. En esta investigación se prefiere en cambio considerar como medida de la falta de inversión pública la dispersión de la población, por el menor interés de los gobiernos en invertir en servicios públicos por los pocos votos esperados a cambio. Es entonces necesario plantearse qué es estar en condición de dispersión, y cómo se mide.

Podrá considerarse en condición de dispersión, tener menos de 14 habitantes por kilómetro cuadrado, la densidad promedio de la Tierra.

En la Figura 2, ofrecida por la Administración Nacional de la Aeronáutica y del Espacio (NASA, por sus siglas en inglés), se presenta un mapa de la densidad poblacional en el mundo.

Para validar si las características de las comunidades marginales se han seleccionado adecuadamente es preciso comprobar que los indicadores de pobreza cuantitativa y cualitativa son peores, que es una cuestión estructural que se hereda generación tras generación y que entre las comunidades los datos son similares, lo que permitiría concluir que en el ámbito rural, los grupos poblacionales en zonas poco aptas para la agricultura y dispersos tienen pocas o ninguna oportunidad para superar la pobreza.

\section{Metodología Para el ANálisis}

Para poder contrastar los indicadores de pobreza cuantitativa y cualitativa con los promedios nacionales se utilizó información primaria obtenida entre 2009 y 2014 en el marco del trabajo desarrollado junto a la Fundación CIDEAL de Investigación y Cooperación, que cede el uso de la información primaria para ser tratada en el presente trabajo.

Los datos de las comunidades se obtuvieron de México, Guatemala, República Dominicana, Haití, Ecuador, Perú, Colombia, Malí, Níger, Senegal y Vietnam, todos países de ingreso medio y bajo, en los que pudieron visitarse 53 comunidades rurales. La población promedio de cada comunidad era de 56 personas. Todas las comunidades estaban en una zona poco apta para la producción agropecuaria, registrándose casos de sabana, bosque tropical perenne, planicie de altura, pronunciada pendiente e inundaciones trimestrales. En todos los casos la dispersión estaba por debajo de los 12 habitantes por kilómetro cuadrado, siendo el promedio de diez habitantes. En la Tabla 1 se indica el detalle de los datos señalados. 
¿Cómo son las comunidades marginales que generan pobreza estructural? /S. HERRERO-OLARTE

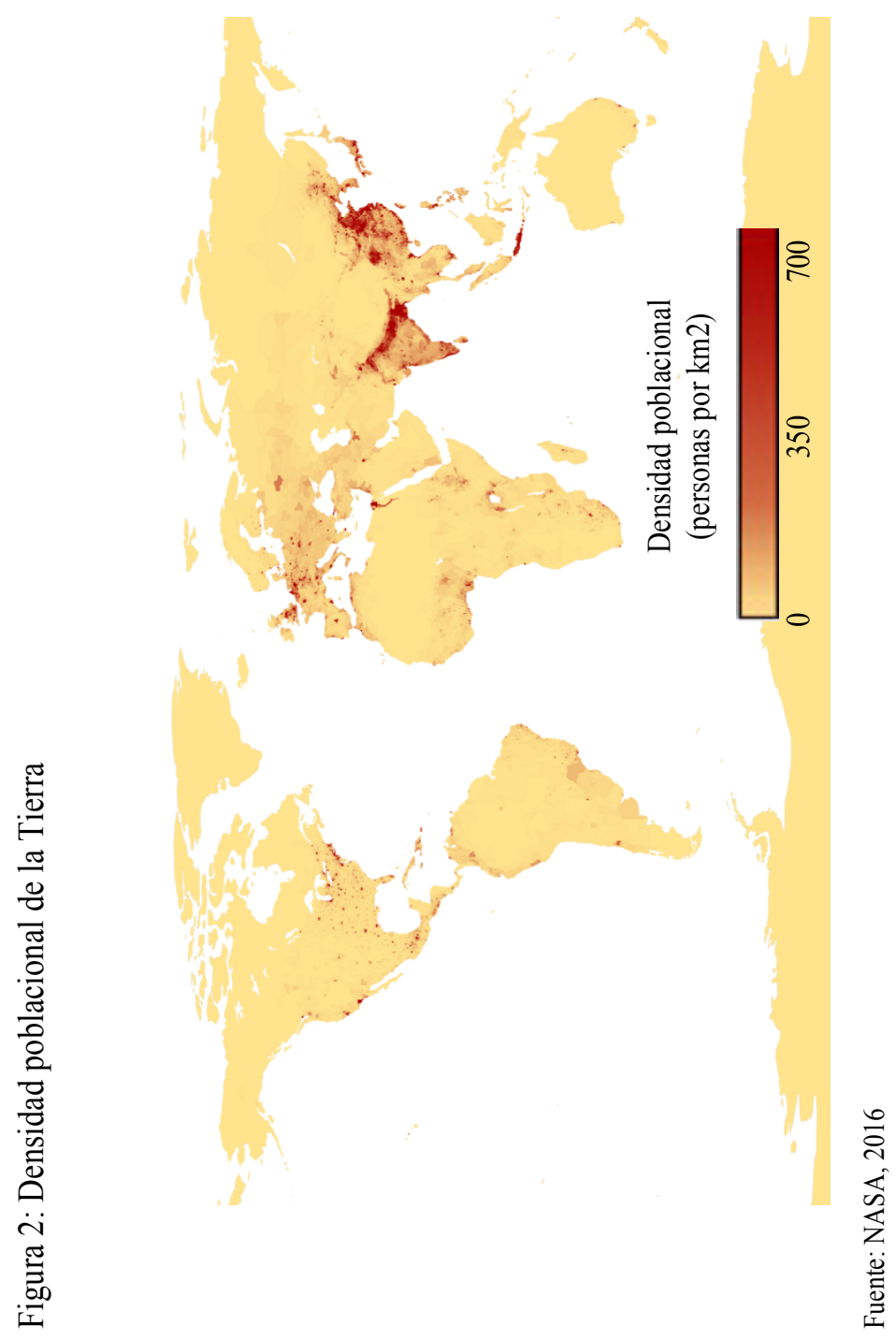




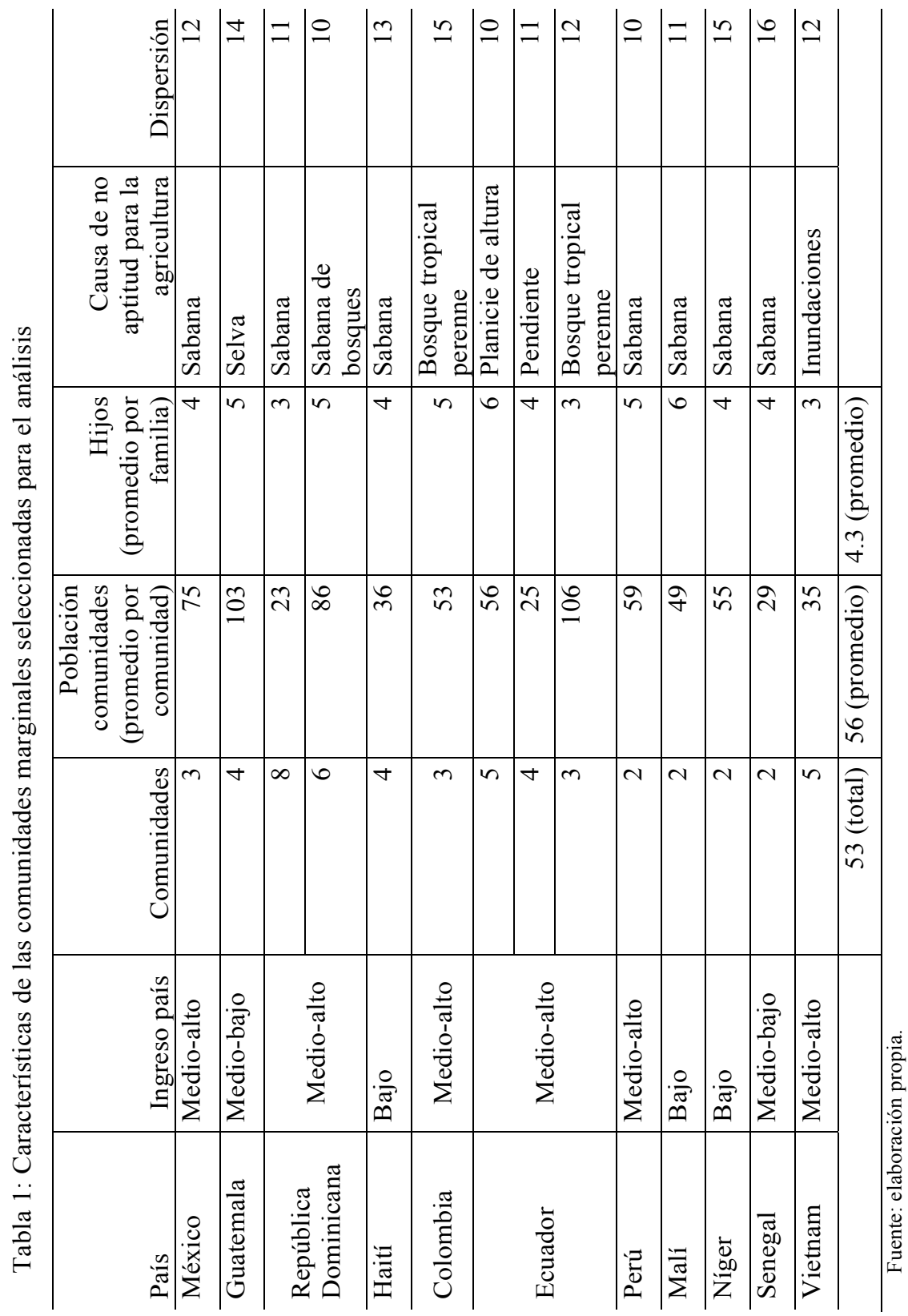


En las comunidades seleccionadas se realizaron 37 entrevistas, diez de ellas en profundidad y se encuestaron 256 personas. Las encuestas se utilizaron para obtener los datos de pobreza en las comunidades y compararlos con los indicadores nacionales y entre las mismas comunidades de los distintos países. Las entrevistas se utilizaron para obtener datos cualitativos sobre la condición de vida de las generaciones anteriores de cara a valorar la condición de estructuralidad y perpetuidad de los datos obtenidos. Más de la mitad eran mujeres. La edad promedio eran 39 años. Se realizaron 23 entrevistas a los directores y/o coordinadores de 13 organizaciones que trabajan en comunidades marginales, y se realizaron 51 encuestas a los técnicos (Tabla 2).

Se identificaron varios indicadores de pobreza cuantitativa y cualitativa y se compararon con las medias nacionales de cada país y el promedio mundial. Los parámetros seleccionados para valorar la pobreza cuantitativa fueron los ingresos. Para el análisis de la pobreza cualitativa, se observaron indicadores de educación, salud y tenencia de bienes, propios del mencionado Índice de Pobreza Multidimensional (IPM).

Para obtener los datos a nivel nacional y mundial de los países seleccionados, si bien se dispone de un volumen de literatura disponible ampliamente analizada, se prefirió contar con una fuente común y homogénea, como es la base de datos del Banco Mundial (Banco Mundial, 2016).

A continuación se presentan los datos comparados de las comunidades y a nivel nacional y mundial de los parámetros seleccionados de pobreza cuantitativa y cualitativa, así como de la persistencia de la pobreza.

\section{INDICADORES DE POBREZA CUANTITATIVA Y CUALITATIVA COMPARADOS}

\section{DE LAS COMUNIDADES MARGINALES}

En la Figura 3 se recogen las diferencias registradas en los datos registrados sobre pobreza cuantitativa. En todos los casos los datos son mejores en el ámbito nacional, al compararlos con los obtenidos en las comunidades de cada país. En cuanto a la cantidad de personas por debajo del umbral de la pobreza los niveles en las comunidades eran muy similares entre sí. La diferencia con los promedios nacionales dependía entonces de las medias del país, lo que indica que los países que han reducido más sus porcentajes de pobreza siguen sin recoger los grupos rurales marginados. En tanto a la tasa de inactividad, la diferencia es similar en todos los casos. 


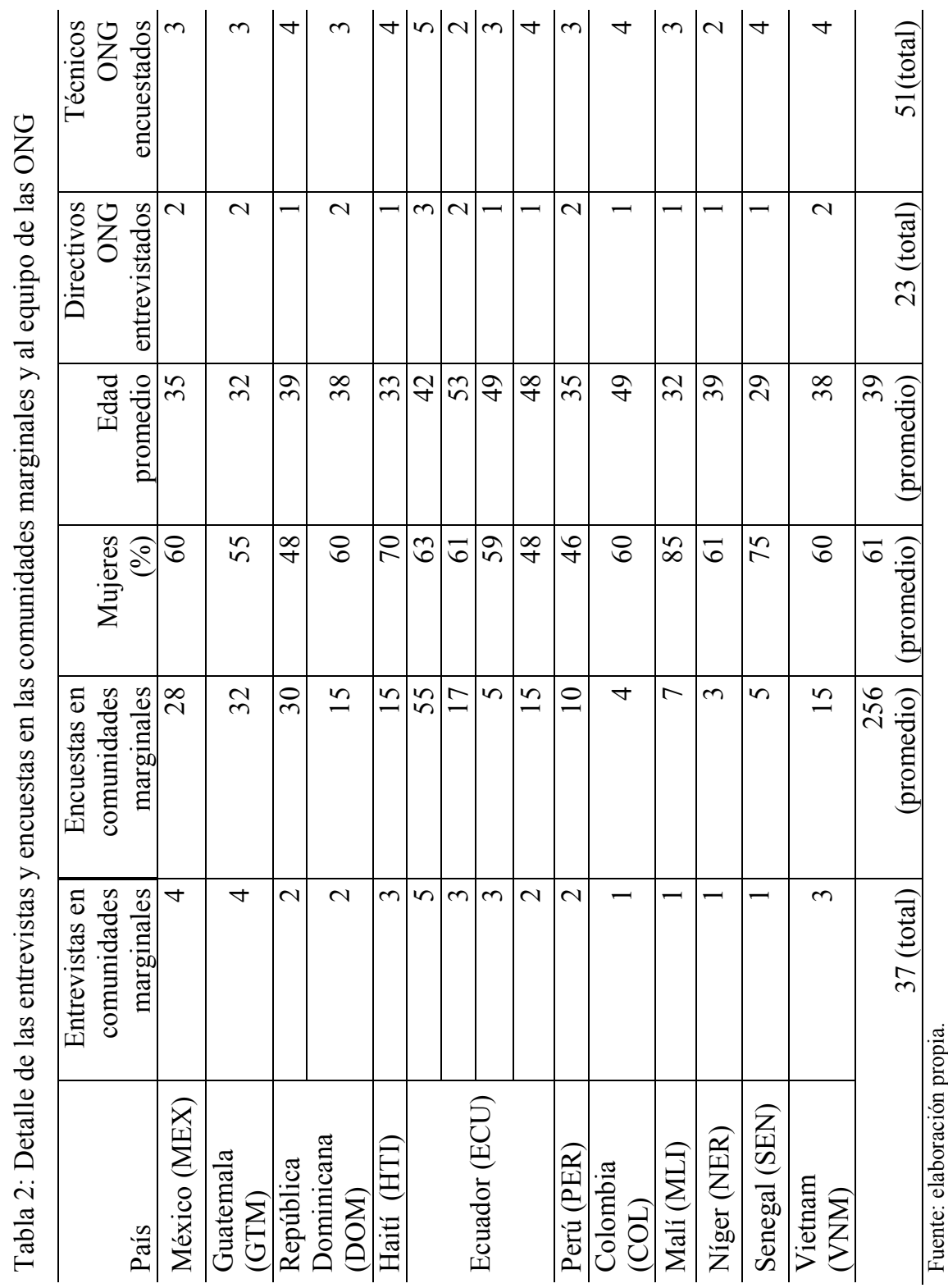


¿Cómo son las comunidades marginales que generan pobreza estructural? /S. HERRERO-OLARTE

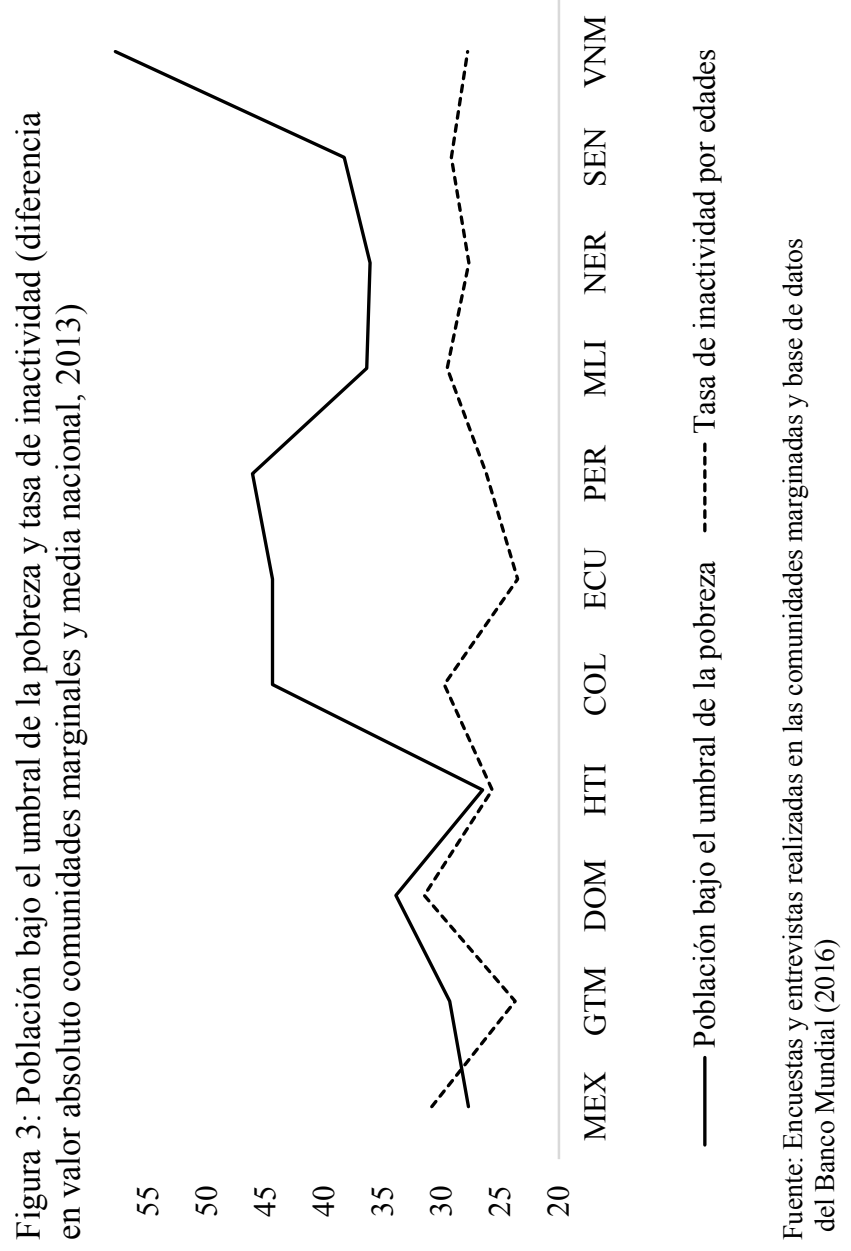


En los países de ingreso bajo como Haití, Malí, Níger y Senegal los porcentajes de personas activas eran especialmente bajos por la escasa productividad de la tierra y las escasas oportunidades laborales, lo que se compensa con los promedios nacionales, que eran también menores. En tanto a los países de ingreso medio, se registran datos en las comunidades igualmente mejores que los de las comunidades de los países de ingreso bajo por las oportunidades laborales que ofrecen los núcleos más cercanos urbanos o rurales con mayor densidad. Como los datos nacionales son también mejores, se registran diferencias similares a las presentadas en los países de ingreso bajo.

Para realizar el análisis cualitativo se han recogido datos de educación, salud y de bienes disponibles. En todos los casos se registran, al igual que en los ejemplos anteriores, mejores datos en el ámbito nacional al compararlos con las medias en las comunidades.

En la Figura 4 se recogen las diferencias registrada en el ámbito de la educación. Al considerar la alfabetización de adultos se presentan las mayores distancias entre los promedios nacionales y de las comunidades en los países que a nivel país tienen los mejores datos, como es el caso de México, Guatemala, Dominicana, Colombia, Ecuador, Perú y Vietnam. El otro grupo de países, formado por Haití, Malí, Níger y Senegal, que presentan los peores niveles a nivel país, no tienen tanta diferencia con los indicadores registrados en las comunidades. Al valorar la diferencia entre la media nacional y en las comunidades de la inscripción escolar en secundaria se aprecia de nuevo una mayor diferencia en el grupo de países que presentan los mejores indicadores, en contraposición de nuevo a los datos presentados en Haití y en los países del África, donde la diferencia es de nuevo mayor. Los datos registrados en las comunidades son similares entre sí, registrándose una diferencia promedio de cinco por ciento entre los mejores indicadores registrados en Ecuador y los peores en Níger.

En la Figura 5 se analizan las condiciones de salud comparadas valorando tres indicadores de referencia como son la prevalencia de la desnutrición considerando las calorías ingeridas y la altura de los niños, y la tasa de mortalidad de los niños.

En cuanto a la desnutrición considerando las calorías ingeridas, no se detectan patrones similares entre grupos de países. En tanto a la prevalencia de la desnutrición se atiende al dato de la altura de los menores de cinco años. Se aprecia como en los países con una mayoría de etnia negra, que presenta promedios generales superiores al resto de grupos poblacionales, como es el caso de la República Dominicana, Haití, Níger y Senegal. 
¿Cómo son las comunidades marginales que generan pobreza estructural? /S. HERRERO-OLARTE

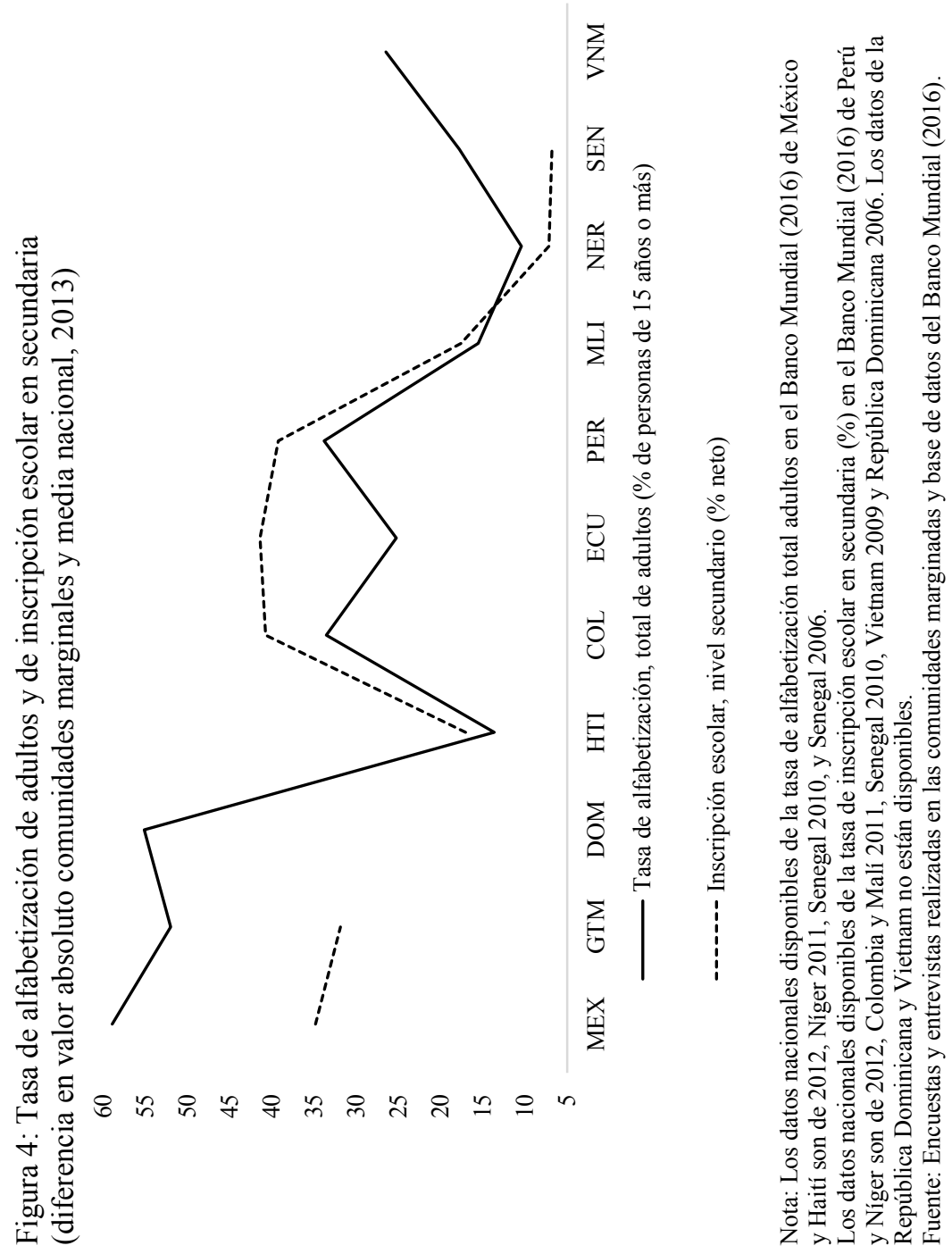




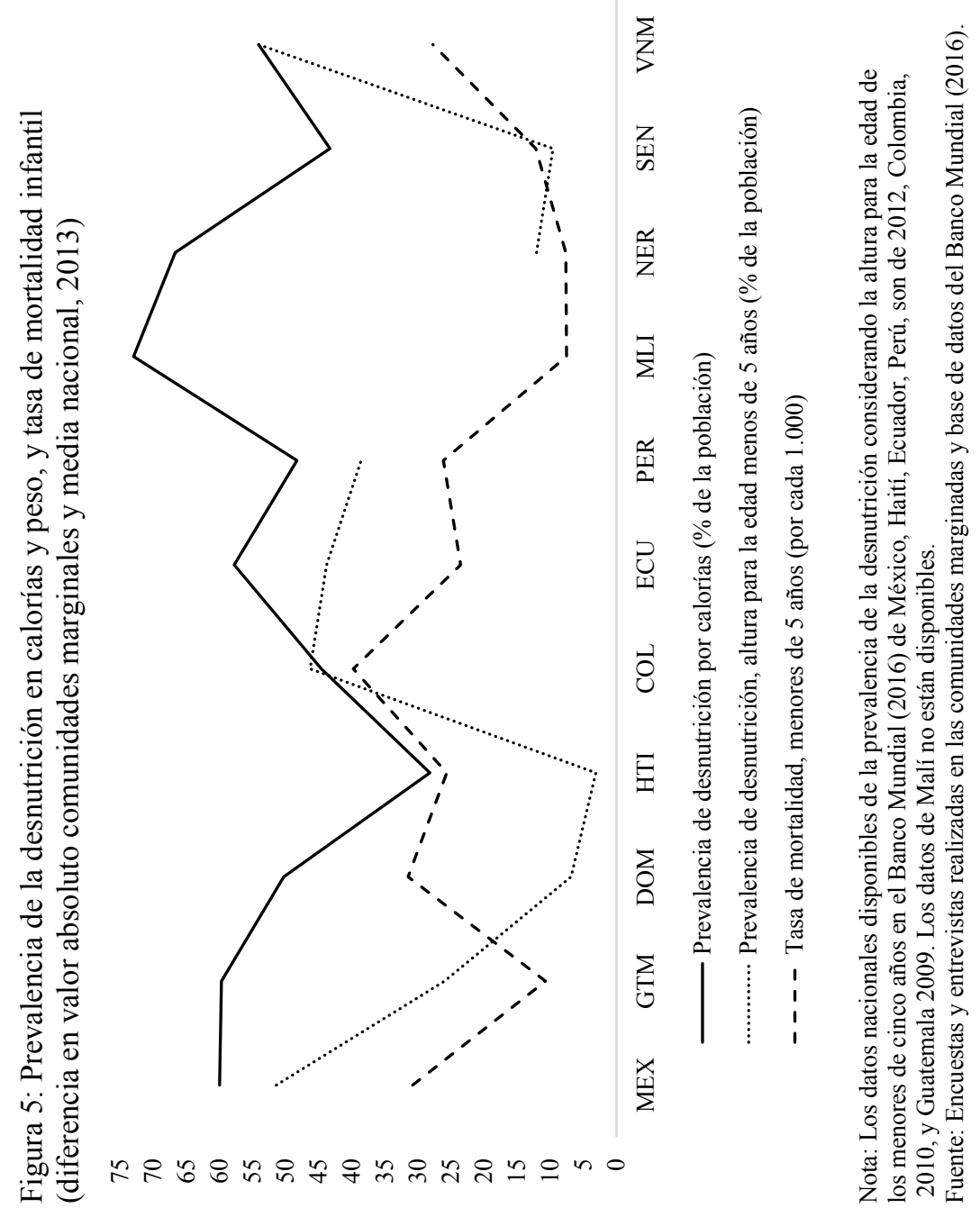


En el resto de países se aprecia una mayor diferencia entre la media nacional y la de las comunidades marginadas porque en éstas suelen residir grupos indígenas con una talla menor que la del resto de etnias. Es preciso entonces considerar si los indicadores antropométricos son los adecuados para valorar la diferencia en las condiciones nutricionales de las comunidades marginales. Al analizar la mortalidad de los menores de cinco años destaca la diferencia entre los países que tienen mejores indicadores de partida, que tienen mayores distancias con las medias de sus comunidades, como es el caso de México, República Dominicana y los tres países andinos considerados.

En la Figura 6, para realizar el análisis de los bienes disponibles, se toman los datos de las instalaciones sanitarias y del acceso a internet. En tanto a las instalaciones sanitarias se aprecia una mayor diferencia entre los datos nacionales y de las comunidades marginales en los países con mejores indicadores nacionales como México, República Dominicana, Colombia, Ecuador y Perú, En cuanto al acceso a internet, en los países que presentan peores datos, como Haití y los africanos, se registra un casi nulo acceso en las comunidades marginadas.

En el análisis de la pobreza, y atendiendo a su dimensión temporal, es preciso analizar hasta qué punto es algo heredado, lo que invitaría a considerar cómo se perpetúan, generación tras generación, indicadores condiciones estructurales que siguen sin superarse. Para ello se atendieron a las entrevistas realizadas en las comunidades a las familias y a los directivos y técnicos de las ONG. En 92 por ciento de los casos los entrevistados consideraban que su condición de vida en general había mejorado ligeramente al compararla con la de las generaciones pasadas, aunque los avances resultaban insuficientes.

Dado que la presente investigación trata de llamar la atención sobre la necesidad de generar políticas de desarrollo específicas en las comunidades marginales para cambiar los indicadores estructurales de pobreza cuantitativa y cualitativa se presenta un análisis que puede ser de utilidad a los titulares de obligaciones.

La visita a las comunidades se aprovechó para analizar las necesidades que las familias y las ONG priorizan para ser atendidas por los responsables. Tal y como se señala en la Figura 7, para 35 por ciento de la ciudadanía el primordial problema era la pobreza, seguido de 16 por ciento, preocupado por la inseguridad alimentaria. Para 36 por ciento, la falta de acceso a sistemas de salud y de educación eran sus principales problemas. 


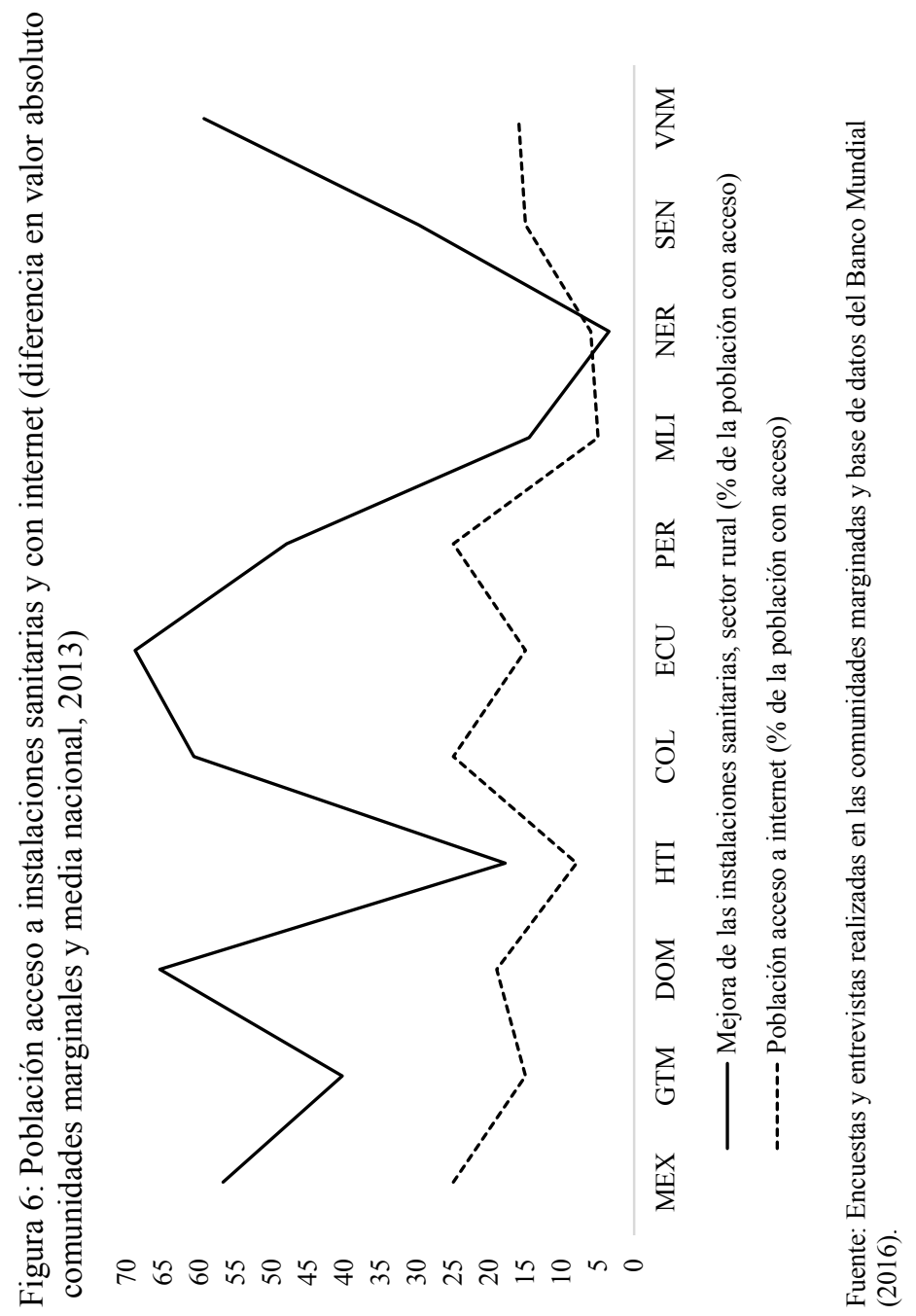


Figura 7: Necesidades detectadas en las comunidades muestra

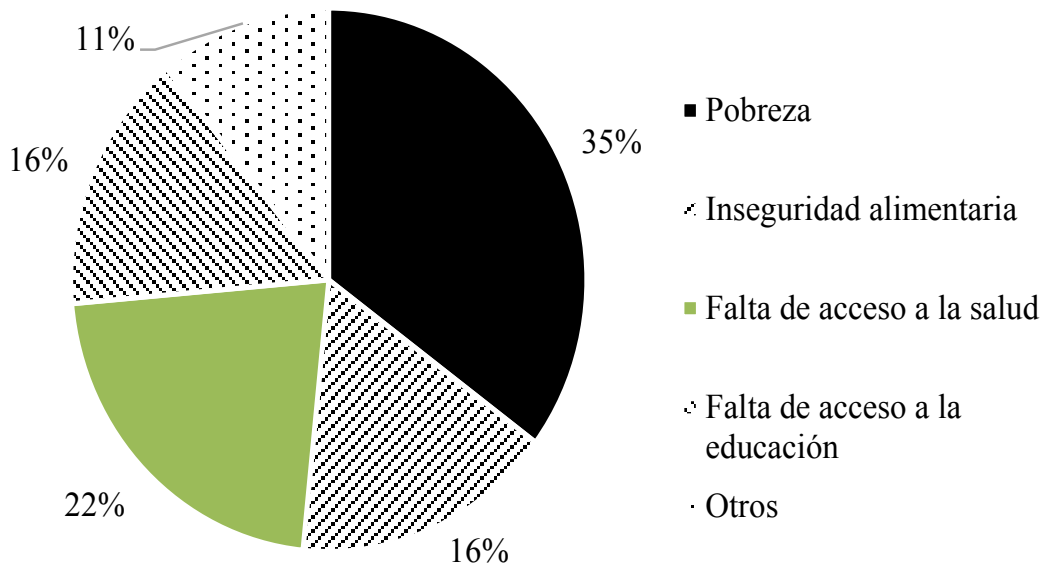

Fuente: Encuestas y entrevistas realizadas en las comunidades marginadas.

\section{Conclusiones}

La pobreza cuantitativa es capaz de mostrar la cantidad de personas que viven con menos dinero del necesario para pagar los gastos necesarios para llevar una vida digna. La pobreza cualitativa considera que el no poder disfrutar de ciertos bienes y servicios, sea como sea que lleguen a la persona, supone estar en condición de pobreza. Las últimas tendencias en el análisis de la pobreza muestran que no son excluyentes entre sí sino más bien complementarias, e invitan a sumar a la valoración de los ingresos otros indicadores para tener una visión global del problema.

El problema es que los mismos indicadores de pobreza pueden registrarse en la periferia de una ciudad, o en una comunidad rural alejada, de manera que la calificación por sí misma no permite considerar las políticas más adecuadas para erradicarla. La definición de exclusión y marginación sí posibilita en cambio el ubicar los espacios físicos en los que reside el mayor porcentaje de personas en condición de pobreza cuantitativa y cualitativa e idear estrategias más viables y eficaces para erradicarla. La exclusión y marginación hacen referencia a la falta de acceso a los canales para generar ingresos, lo que perpetúa la pobreza cuantitativa, y a los servicios básicos, lo que se relaciona con la pobreza cualitativa. Lo difícil es definir qué es estar en condición de exclusión, concepto relacionado con ámbito urbano, o de marginación, en el campo. 
Atendiendo a la marginación, e invitando a la consideración de la exclusión en otro trabajo de investigación, se acota la definición al ámbito rural de los países de renta media y baja. Si no tienen acceso a los canales para incrementar sus recursos, es porque una fuente de ingresos fundamental, como es la agricultura, no ofrece los elementos necesarios para alcanzar los niveles óptimos de productividad. Esto se da cuando la comunidad se ubica en zonas poco aptas para la producción agropecuaria como son los desiertos, las zonas de elevada pendiente, la selva, etc. La escasa inversión pública coincide con las comunidades en el campo en el que el grado de dispersión es elevado porque el resultado en cuanto a los votos que se recibirán después resulta insuficiente.

La definición de comunidad marginada es entonces la de aquélla ubicada en el ámbito rural en un país de ingreso medio o bajo, en una zona poco apta para la producción agropecuaria y con un elevado nivel de dispersión poblacional.

Es preciso valorar la pobreza cuantitativa y cualitativa en las comunidades con estas características para concluir si, como se espera, los indicadores son especialmente desfavorables. Además es necesario comprobar si las condiciones de vida han sido similares para las generaciones anteriores, lo que permitiría pensar que se trata de una cuestión estructural. Es preciso considerar también si los datos son similares entre las comunidades, para valorar si condiciones de vida similares generan resultados en términos de pobreza cuantitativa y cualitativa parecidos. Para realizar en análisis se compararon indicadores de pobreza cuantitativa y cualitativa en comunidades marginadas de once países identificadas según la definición propuesta con el promedio nacional de cada país.

El resultado muestra cómo, en todos los casos, los niveles registrados en las comunidades de todos los indicadores han sido sustancialmente peores a los registrados a nivel país. Los indicadores de pobreza cuantitativa respeto a la media nacional son 56 por ciento más elevados, mientras los que la pobreza cualitativa son 35 por ciento más altos. Los resultados fueron ligeramente peores para las generaciones anteriores lo que permite considerar que en las comunidades marginales se hereda una pobreza estructural que es especialmente difícil cambiar. La actuación en las comunidades marginales, a tenor de los datos obtenidos, debería resultar prioritaria para los titulares de obligaciones relacionados.

El análisis realizado muestra una similitud general de los datos entre las comunidades, independientemente del país y del contexto, especialmente de pobreza cuantitativa. Es posible entonces considerar la posibilidad de 
definir una estrategia marco para generar ingresos en estas comunidades que pueda considerarse para impulsar las políticas de desarrollo nacional y regional de las comunidades marginadas. La estrategia marco podría servir para el análisis a nivel país del tratamiento de la pobreza en las comunidades marginadas bien sea para utilizarla, adaptarla o desecharla pero serviría de base para tratar una cuestión que todavía los titulares de obligaciones no saben cómo abordar. Las necesidades que han priorizado las familias y las ONG en las comunidades marginales para su consideración en la estrategia a definir deberían abordar la falta de ingresos, la inseguridad alimentaria y la falta de acceso a un sistema de salud y educación de calidad.

Profundizando en los datos, al considerar los indicadores de pobreza cualitativa se obtienen mejores resultados en los países de ingreso medio fruto de las políticas desarrolladas de acceso a servicios básicos de salud y educación de las que en cierta medida, aunque todavía tímidamente, han podido beneficiarse en las comunidades. Los datos absolutos son coherentes con los comparados. En efecto, la diferencia entre los indicadores registrados de pobreza cualitativa en las comunidades y a nivel nacional es mayor en los casos de los países de ingreso medio, lo que invita a pensar que en los procesos de desarrollo seguidos, aunque todavía inconclusos, no han formado parte las comunidades marginadas. Es posible entonces pensar en si la estrategia a seguir por resultados para erradicar la pobreza cualitativa en las comunidades marginadas debe tratar de alcanzar un desarrollo concentrado en ciertos polos que atraigan a parte de la ciudadanía de las comunidades o en si es mejor llevar los servicios hasta las comunidades mismas.

\section{REFERENCIAS BIBLIOGRÁFICAS}

Abrahamson, Peter, 1997, Exclusión social en Europa: ¿vino viejo en odres nuevos?, Madrid. CSIC.

Altimir, Oscar, 1979, La dimension de la pobreza en America Latina, Cuadernos de CEPAL, 27, CEPAL, Santiago de Chile.

Arriba, Ana, 2002, El concepto de exclusión en política social. Unidad de Políticas Comparadas. España. CSIC: Documento de Trabajo 02-01. Recuperado de http:// www.iesam.csic. es/doctrab2/dt-0201.pdf.

Bamberger, Michael, 2000, Integrating quantitative and qualitative research in development projects. Washington D.C.World Bank Publications.

Banco Mundial, 2016, Base de datos, Washington: BM. Disponible en http://datos.bancomundial.org/ (Consultado el 15/06/2012). 
Bárcena, Martin, 2001, Privación relativa, bienestar e imposición sobre la renta. Tesis Doctoral. Málaga. Universidad de Málaga.

Berrebi, Joseph y Silber, Jacques, 1985, "Income inequality indices and deprivation: A generalization", en Quarterly Journal of Economics, Oxford.

Bowen, Glenn, 2009, "Document analysis as a qualitative research method. Carolina Del Norte", en Qualitative research journal, 9(2), 27-40.

Booth, Charles, 1889, Life and labor of the people, vol. 1, Williams and Norgate.

Cardoso, Fernando, 1970, "Comentario sobre los conceptos de sobrepoblación relativa y marginalidad", en Revista Latinoamericana de Ciencias Sociales, núms. 1 y 2. Santiago de Chile.

Castel, Robert, 1998, "La lógica de la exclusión" Bustelo, Eduardo y Minujin, Alberto, Propuesta para sociedades incluyentes. Colombia. Unicef/Cuadernos Santillana.

Chakravarty, Satya y Mukherjee, D., 1998, "Lorenz domination, utilitarian deprivation rule and equal sacrifice principle", en The Manchester School Journal 66, $5,521-531$.

Chakravarty, Satya y Moyes, Patrick, 2002, Individual welfare, social deprivation and income taxation. India. Economic Theory.

Charles Booth, 1889, Labour and life of the people in London, vol. 1. East London. London: Williams and Norgate.

Davis, James, 1959, "A formal interpretation of the Theory of the relative Deprivation", en Sociometry. 20. 280-296.

Desal, 1969, "La marginalidad en América Latina: un ensayo de diagnóstico", en Cuadernos de Relaciones Laborales. 23-2. 247-267, Barcelona. Herder.

Ebert, Udo y Moyes, Patrick, 2000, “An axiomatic characterization of Yitzhaki's index of individual deprivation", en Economics Letters 68, 263-270

Fromm, Erich, 1956, Psicoanálisis de la sociedad contemporánea. México. Fondo de Cultura Económica.

Geremek, Bronislaw, 1991, Les fils de Caïn. París. Flammarion.

Giusti, Jorge, 1973, Organización y participación popular en Chile: el mito del hombre marginal. Buenos Aires. Ediciones Flacso.

Glaser, Barney y Strauss, Anselm, 1967, The discovery of grounded theory: strategies for qualitative research. New York. Aldine Publishing Company.

Golovensky, Vstore, 1951-1952, "The marginal man concept", en Analysis and Critique. vol. 30.

Green, A. W., 1947-1948, “A Re-examination of the Marginal Man Concept", en Social Forces, vol. 26, 1947-1948.

Heilbroner, Robert, 1976, "Business Civilization", en Decline by Robert. New York. W. W. Norton y Company. 
Heller, Agnes, 1978, Teoría de las necesidades en Marx. Barcelona. Península.

Henrich, Joe et al., 2005, "Economic man" in cross-cultural perspective: Behavioral experiments in 15 small-scale societies. Atlanta, USA. Department of Anthropology, Emory University, Atlanta.

Hey, Jhon y Lambert, Peter, 1980, "Relative deprivation and the Gini coefficient: Comment", en Quarterly Journal of Economics 95, 567-573. Cambridge.

Imedio, Luis y Barcena, Elena, 2002, Privación status e imposición sobre la renta. Estudios de Economía Aplicada. España. Asociación internacional de Economía aplicada.

Kovacevic, Milorad y Calderon, Cecilia, 2014, UNDP's Multidimensional Poverty Index. Specifications. UNDP.

Lenoir, Rene, 1974, Les exclus. París. Le Seuil.

Malthus, Thomas, 1846, Ensayo sobre el principio de la población. Madrid. Est. Lit. y Tip. de Lucas Gonzalez y Compañía. Universidad Complutense de Madrid

Márkus, Gyorgy, 1974, Marxismo y Antropología. Barcelona. Grijalbo.

Maslow, Abraham, 1943, “A theory og Human Motivtion”, en Psychological Review, vol. 50. Toronto. York University.

Massé, Pierre, 1965, L’exclusion sociale. París. Klenfer.

Merton, Robert, 1938, "Social Structure and Anomie", en American Sociological Review 3. United States. vol 3, Issue 5. 672-682

Merton, Robert y Kitt, A.S., 1950, Contributions to the Theory of Reference Group Behavior. New York. Glencoe, Ill.: Free Press. Pages 40-105.

Minujin, Alberto, 1998, "Vulnerabilidad y exclusión social en América Latina", en Bustelo, Eduardo y Minujin, Alberto, Todos entran: propuesta para sociedades incluyentes. Colombia. Unicef/Cuadernos Santillana.

Moreno, Luis, 2000, Ciudadanos precarios, la última red de protección social. Barcelona. Ariel.

Mukherjee, D., 1997, "Deprivation reducing income tax functions", en Research on economic inequality, vol. 7, 153-163.

NASA, 2016, Densidad poblacional. Disponible en http://visibleearth.nasa.gov/ view.php?id=53005, (Consultado el 29/087/2013).

Olarte, Susana Herrero, 2017, "El rol de la sociedad civil en el reconocimiento de la seguridad alimentaria como bien público regional en Latinoamérica", en Revista Aportes para la Integración Latinoamericana, (36).

Park, R., 1928, "La migración humana y el hombre marginal” vol. 33 of American Journal of Sociology.

Podder, Nripesh, 1996, "Relative deprivation, envy and economic inequality", en Kyklos 49, 353-376. 
Pradhan, Menno y Ravallion, Martin, 2000, "Measuring poverty using qualitative perceptions of consumption adequacy", en Review of Economics and Statistics, Washington. 82(3), 462-471.

Raya, Esther, 2005, Categorías sociales y personas en situación de exclusión. Una aproximación. Madrid. Universidad Complutense de Madrid. Recuperado de http://www.redalyc.org/pdf/112/11204704.pdf

Renes, Victor, 1993, Luchar contra la pobreza hoy. Madrid. HOAC.

Ravallion, Martin, 1998, Poverty Lines in theory and practice. Living Standards Measurentent Study. Working paper núm. 133. Washington. Banco Mundial.

Ricardo, David, 1986, On the Principles of Political Economy and Taxation. Editado por Piero Sraffa. Cambridge. Cambridge University Press.

Runciman, W., 1966, Relative deprivation and social justice. Londres. Routledge and Kegan Paul.

Sebastian, Kate, 2009, Mapping favorability for agriculture in low and middle income countries: technical report, maps and statistical tables. Washington. Oxfam América.

Sen, Amartya, 1982, Utilitarianism and beyond. Cambridge. Cambridge University Press.

Sen, Amartya and Dreze, J., 1990, The political economy of hunger. Oxford. Oxford: Clarendon Press.

Sen, Amartya, 1985, Commodities and Capabilities. Amsterdam. North-Holland. Oxford University Press.

Sen, Amartya, 1984, Resources, values and development. Oxford. Basil Blackwell.

Sen, Amartya, 1999, Development as Freedom. Oxford. Oxford University Press.

Smael, M., y Alpert, E., 2009, Making investments in poor farmers pay: A review of evidence and sample of options for marginal areas. Washington. Oxfam América.

Stouffer S.A.; Suchman, E.A.; Devinney, L.C.; Star, S.A.; y Williams, R.M., 1949, The American soldier: Adjustment during army life 1. New Jersey. Princeton University Press. Princeton NJ.

Streeten, Paul; Javed, Burki; Mahbub ul Haq; Hicks, Norman y Stewart, Frances, 1981, First things first: meeting basic human needs in developing countries. New York and Oxford. Oxford University Press.

Tezanos, Jose Felix, 1999, Tendencias en desigualdad y exclusión social. Tercer foro sobre tendencias sociales. Madrid. Sistema.

Thaler, Richard, 1988, “Anomalies: The Winner's Curse”, en Journal of Economic Perspectives, 2(1): 191-202. DOI: 10.1257/jep.2.1.191. 
Thomas, Willian y Znaniecki, Florian, 1918, El campesino polaco en Europa y en América. Boston. Bagder.

Townsend, Peter, 1979, Poverty in the United Kingdom. Reino Unido. Prenguin, Harmondsworth.

UNESCO, 1960, Convención relativa a la Lucha contra las Discriminaciones en la Esfera de la Enseñanza de 1960. Recuperado de http:/www.unesco.org/filead$\mathrm{min} / \mathrm{MULTIMEDIA} / \mathrm{HQ} / \mathrm{ED} / \mathrm{GMR} / \mathrm{pdf} / \mathrm{gmr} 2010 / \mathrm{gmr} 2010$-ch3-es.pdf

Wiggins, David, 2002, Needs, Values, Truth. Essays in the Philosophy of Value. Oxford, Reino Unido. Clarendon Press.

Yitzhaki, Shlomo, 1982, "Relative deprivation and economic welfare", European Economic Review 17, 99-113.

Yitzhaki, Shlomo, 1979, "Relative deprivation and the Gini coefficient", en Quarterly Journal of Economics 93, 321-324. Cambridge.

\section{RESUMEN CURRICULAR DE LA AUTORA}

Doctora en Economía Aplicada. Coordinadora del Centro de Investigaciones Económicas y catedrática de economía en Universidad de Las Américas (UDLA) en Quito, Ecuador. Consultora para organismos nacionales e internacionales en Latinoamérica, África y Asia.

ORCIDID: https://orcid.org/0000-0003-3509-6316

Dirección electrónica: olartesusana@hotmail.com

Artículo recibido el 15 de abril de 2016 y aprobado el 4 de abril de 2018 . 